\title{
Perfil clínico-epidemiológico de pacientes infectados pelo HTLV-1 em Belém/Pará
}

\author{
Clinical-epidemiological profile of HTLV-1 infected patients \\ in Belém, Pará state, Brazil
}

\author{
Luzielma Macêdo Glória¹, Suzielle de Arruda Damasceno", Luana Rego Rodrigues", \\ Mayara do Socorro Brito dos Santos ${ }^{1}$, Rita Medeiros², George Alberto da Silva Dias², \\ Denise da Silva Pinto 4
}

\begin{abstract}
Resumo
Objetivo: Investigar o perfil clínico-epidemiológico de pacientes portadores do HTLV-1 em Belém, no Estado do Pará, Brasil, descrevendo as características de indivíduos infectados e atendidos no Laboratório de Clínica e Epidemiologia de Doenças Endêmicas (LCEDE) do Núcleo de Medicina Tropical (NMT) da Universidade Federal do Pará (UFPA). Metodologia: Foi um estudo descritivo de corte transversal, com coleta em prontuários de pacientes infectados pelo HTLV-1, matriculados no LCEDE do NMT da UFPA entre os anos 2000 a 2012. Foi aplicada a análise descritiva pelo programa Epi Info 3.2.5. Resultados: Dos 182 prontuários, $35,2 \%$ eram do sexo masculino, e $64,8 \%$, do sexo feminino. Houve predomínio de indivíduos casados (47,8\%). A maioria $(73,6 \%)$ tinha procedência do município de Belém-PA. Dentre os pacientes sintomáticos, $51 \%$ apresentavam sintomas neurológicos, $21 \%$, queixas dermatológicas, $18 \%$, queixas reumatológicas, e 10\%, queixas autonômicas. Com relação aos pacientes com sintomas neurológicos, 16 (39,0\%) foram diagnosticados com PET/MAH. Conclusões: Houve o predomínio do sexo feminino, na faixa etária entre 40 e 49 anos, casado, trabalho informal, procedentes de Belém, com queixas neurológicas e com retorno regular as consultas médicas.
\end{abstract}

Palavras-chave: epidemiologia; infecção pelo vírus linfotrópico de células T humana 1; Belém.

\begin{abstract}
Objective: The clinical-epidemiological profile of patients with HTLV-1 in Belém, Pará state, Brazil was investigated describing the characteristics of these patients at an outpatient service of the Clinical and Epidemiologic Laboratory of Endemic Diseases - LCEDE from the Tropical Medicine Center - NMT of the Federal University of Pará - UFPA. Methodology: It was a descriptive, cross-sectional study with data collected from the records of HTLV-1 patients from 2000 to 2012. Descriptive analysis used Epi Info 3.2 .5 software. Results: Of the 182 patients, $35.2 \%$ were male and $64.8 \%$ female, with a predominance of married individuals (47.8\%), approximately $73.6 \%$ were from Belém. Among the symptomatic patients, $51 \%$ presented neurological symptoms, $21 \%$ dermatological complaints, $18 \%$ rheumatologic complaints, and $10 \%$ autonomic complaints. Among the patients with neurological symptoms, 16 (39\%) were diagnosed with HAM/TSP. Conclusion: There is predominance of females, aged 40-49 years, married, with informal jobs, from Belém, with neurological sings and regular return to medical visits.
\end{abstract}

Keywords: epidemiology; human T cell lymphotropic virus type 1 infection; Belém.

\footnotetext{
${ }^{1}$ Acadêmicas do curso de Fisioterapia da Faculdade de Fisioterapia e Terapia Ocupacional (FFTO) da Universidade Federal do Pará (UFPA) - Belém (PA), Brasil. 2 Doutora em Virologia pela Universidade Paris 7 - Instituto Pasteur. Professora do Programa de Pós-graduação em Doenças Tropicais pelo Núcleo de Medicina Tropical (NMT) da Universidade Federal do Pará (UFPA) - Belém (PA), Brasil.

${ }^{3}$ Doutor em Doenças Tropicais pelo Programa de Pós-graduação em Doenças Tropicais do Núcleo de Medicina Tropical (NMT). Professor Assistente da Faculdade de Fisioterapia e Terapia Ocupacional (FFTO) da Universidade Federal do Pará (UFPA) - Belém (PA), Brasil.

${ }^{4}$ Doutora em Doenças Tropicais pelo Núcleo de Medicina Tropical. Professora Adjunta III da Faculdade de Fisioterapia e Terapia Ocupacional (FFTO) da Universidade Federal do Pará (UFPA) - Belém (PA), Brasil.

Trabalho realizado na Faculdade de Fisioterapia e Terapia Ocupacional (FFTO) da Universidade Federal do Pará (UFPA) - Belém (PA), Brasil. Endereço para correspondência: George Alberto da Silva Dias - Rua Augusto Corrêa, 01 - Guamá - CEP: 66075-110, Belém (PA), Brasil -

Email: georgealbertodias@yahoo.com.br

Fontes de financiamento: nenhuma.

Conflito de interesses: nada a declarar.
} 


\section{INTRODUÇÃO}

O vírus linfotrópico de células T humanas do tipo 1 (HTLV-1) é classificado como um complexo retrovírus do tipo C, pertencente ao gênero Deltaretrovirus da família Retroviridae e da subfamília

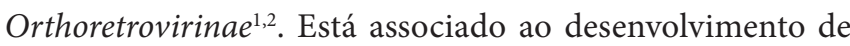
doenças graves, tais como a leucemia/linfoma de células $\mathrm{T}$ do adulto (LLcTA) e a paraparesia espástica tropical/mielopatia associada ao HTLV-1 (PET/MAH), além de inúmeras doenças correlacionadas com a infecção, por exemplo: a uveíte, síndrome de Sjögren, dermatite infecciosa, polimiosite, artropatias, tireoidite, polineuropatias, alveolite linfocitária, linfoma cutâneo de células T, estrongiloidíase, escabiose, hanseníase e tuberculose ${ }^{3-6}$.

O HTLV-1 tem ampla distribuição mundial e estima-se que cerca de 15 a 20 milhões de pessoas estejam contaminadas. Muitas são as áreas endêmicas para o vírus, como o sul do Japão, o Caribe, a América Central e do Sul, o Oriente Médio, a África, as Ilhas da Melanésia, além de três focos pequenos encontrados na população aborígene da Austrália, na Papua Nova Guiné e no norte do Japão $0^{6-11}$.

No Brasil, a infecção pelo HTLV-1 é considerada endêmica. Estimativas apontam que aproximadamente 2,5 milhões de pessoas estejam infectadas pelo vírus, o que torna o Brasil o país com maior número absolutos de casos. Essa alta prevalência varia entre as regiões brasileiras, apresentando baixa ocorrência na região Sul do país e alta nas regiões Norte e Nordeste ${ }^{12-14}$.

A transmissão do HTLV-1 ocorre, principalmente, por três vias. A primeira via, chamada de sexual, é considerada a menos eficiente, no entanto a transmissão do vírus do homem para mulher ocorre cerca de quatro vezes mais quando comparada à transmissão da mulher para o homem. Na segunda via, chamada de perinatal, ocorre a transferência de linfócitos maternos infectados para o neonato, especialmente durante o processo de amamentação, sendo considerada a via mais frequente. Por fim, a terceira via chamada de sanguínea, representa a forma mais eficiente de transmissão do vírus, ocorrendo por meio de transfusões sanguíneas, transplante de órgãos ou pelo uso de fômites ${ }^{8,15-18}$.

O diagnóstico da infecção pelo HTLV-1 baseia-se no rastreio sorológico de anticorpos específicos, por intermédio de ensaio imunoenzimático (ELISA) e do Western blot, além de testes moleculares confirmatórios, como a reação em cadeia da polimerase (PCR) ${ }^{19}$.

Apesar de a região Norte do país apresentar elevada prevalência para o HTLV-1, estudos envolvendo o perfil epidemiológico em Belém, no Estado do Pará, Brasil, ainda são escassos. O desconhecimento do vírus e as doenças causadas por ele dificultam o rastreio populacional. Este estudo teve como objetivo investigar o perfil clínico e epidemiológico de pacientes portadores do HTLV-1 em Belém-PA, descrevendo as características de indivíduos infectados e atendidos no Laboratório de Clínica e Epidemiologia de Doenças Endêmicas (LCEDE) do Núcleo de Medicina Tropical (NMT) da Universidade Federal do Pará (UFPA).

\section{METODOLOGIA}

Trata-se de um estudo descritivo de corte transversal, no qual foram incluídos 408 prontuários de pacientes infectados pelo HTLV-1, matriculados no LCEDE do NMT da UFPA entre os anos 2000 a 2012. O estudo foi aprovado pelo Comitê de Ética em Pesquisa do NMT sob Parecer no 063/2011.

Os dados dos prontuários foram coletados em formulário-padrão, incluindo as seguintes variáveis: idade, sexo, profissão, estado civil, procedência, morbidades associadas ao HTLV-1 e a realização de acompanhamento clínico.

As morbidades relacionadas ao vírus foram coletadas por meio das informações contidas nos prontuários preenchidas pelos médicos responsáveis pelo acompanhamento clínico desses pacientes. Para as queixas neurológicas, foram coletadas informações acerca da espasticidade e da fraqueza muscular em membros inferiores, além de alterações na sensibilidade e nos reflexos. Outro aspecto relacionado a alterações neurológicas e que foram classificados como queixas autonômicas eram as alterações nos esfíncteres anal e vesical. Para as alterações dermatológicas, foram coletadas informações sobre a presença de escabiose, dermatite seborreica, dermatofitoses, xerose e dermatite infecciosa. Para as alterações reumatológicas, foram observadas informações sobre a presença de síndrome de Sjögren, artrite reumatoide, lúpus eritematoso sistêmico, artropatia relacionada ao vírus, osteoartrite e manifestação articular de doença sistêmica. Todas estas alterações (neurológicas, dermatológicas, reumatológicas e autonômicas) podem estar relacionadas ao HTLV-1, sendo as neurológicas as mais importantes, pois podem se relacionar com o desenvolvimento da PET/MAH.

Foram incluídos no estudo todos os indivíduos infectados exclusivamente pelo HTLV-1, sintomáticos ou não, com exclusão de prontuários de indivíduos coinfectados por outros patógenos, sem especificação do tipo de HTLV e sem a confirmação laboratorial da infecção viral por testes moleculares. O diagnóstico da PET/MAH seguiu os critérios propostos pela $\mathrm{OMS}^{20}$ e também foi utilizado o novo critério de diagnóstico proposto por Castro-Costa et al. ${ }^{20}$.

Para análise estatística das características clínica e epidemiológica, as variáveis categóricas foram apresentadas como frequências, e as numéricas, por meio de medidas de tendência central e de dispersão, sendo calculado intervalo de confiança a $95 \%$ para todas as categorias. Todo o processamento estatístico foi realizado nos softwares Epi Info version 3.5.2. 


\section{RESULTADOS}

Dos 408 prontuários consultados inicialmente, foram excluídos 226 dos quais 118 por apresentarem coinfecção por outros patógenos e 108 por apresentarem testes moleculares com resultados inconclusivos ou sem resultados, permanecendo 182 prontuários viáveis para análise final deste estudo.

Tabela 1. Caracterização dos pacientes infectados pelo HTLV-1 em Belém/Pará

\begin{tabular}{|c|c|c|c|}
\hline Variáveis & $\mathbf{n}$ & $\%$ & IC $95 \%$ \\
\hline \multicolumn{4}{|l|}{$\operatorname{Sexo}(N=182)$} \\
\hline Masculino & 64 & 35,2 & $28,2-42,6$ \\
\hline Feminino & 118 & 64,8 & $57,4-71,8$ \\
\hline \multicolumn{4}{|l|}{ Idade $(\mathrm{N}=182)$} \\
\hline Média \pm Desvio Padrão & \multicolumn{2}{|c|}{$43,7 \pm 12,8$ anos } & $41,8-45.5$ \\
\hline Mediana & \multicolumn{2}{|c|}{44 anos } & \\
\hline \multicolumn{4}{|l|}{ Faixa etária $(\mathrm{N}=182)$} \\
\hline 10 a 19 anos & 3 & 1,7 & $0,3-4,7$ \\
\hline 20 a 29 anos & 28 & 15,4 & $10,5-21,5$ \\
\hline 30 a 39 anos & 35 & 19,2 & $13,8-25,7$ \\
\hline 40 a 49 anos & 52 & 28,6 & $22,1-35,7$ \\
\hline 50 a 59 anos & 46 & 25,3 & $19,1-32,2$ \\
\hline 60 a 69 anos & 13 & 7,1 & $3,9-11,9$ \\
\hline 70 a 79 anos & 4 & 2,2 & $0,6-5,5$ \\
\hline 80 a 89 anos & 1 & 0,6 & $0,0-3,0$ \\
\hline \multicolumn{4}{|l|}{ Estado civil $(\mathrm{N}=182)$} \\
\hline Casado & 87 & 47,8 & $40,4-55,3$ \\
\hline Divorciado & 6 & 3,3 & $1,2-7,0$ \\
\hline Solteiro & 76 & 41,7 & $34,5-49,3$ \\
\hline Viúvo & 13 & 7,2 & $3,9-11,9$ \\
\hline \multicolumn{4}{|l|}{ Profissão (N=182) } \\
\hline Trabalho formal & 66 & 36,2 & $29,3-43,7$ \\
\hline Trabalho informal & 88 & 48,4 & $40,9-55,9$ \\
\hline Aposentado & 14 & 7,7 & $4,3-12,6$ \\
\hline Estudante & 14 & 7,7 & $4,3-12,6$ \\
\hline \multicolumn{4}{|l|}{ Procedência $(\mathrm{N}=182)$} \\
\hline Belém & 134 & 73,6 & $66,6-79,9$ \\
\hline Região Metropolitana & 34 & 18,7 & $13,3-25,1$ \\
\hline Interior do Estado & 13 & 7,2 & $3,9-11,9$ \\
\hline Outros Estados & 1 & 0,5 & $0,0-3,0$ \\
\hline \multicolumn{4}{|l|}{ Sintomatologia $(\mathrm{N}=182)$} \\
\hline Assintomáticos & 102 & 56,0 & $48,5-63,4$ \\
\hline Sintomáticos & 80 & 44,0 & $36,6-51,5$ \\
\hline \multicolumn{4}{|c|}{ Manifestações clínicas $(\mathrm{N}=\mathbf{8 0})$} \\
\hline Neurológicas & 41 & 51,0 & $40,3-62,2$ \\
\hline Dermatológicas & 17 & 21,0 & $12,3-30,2$ \\
\hline Reumatológicas & 14 & 18,0 & $9,2-25,8$ \\
\hline Autonômicas & 8 & 10,0 & $3,4-16,6$ \\
\hline \multicolumn{4}{|c|}{ Manifestações neurológicas $(\mathrm{N}=41)$} \\
\hline Com PET/MAH & 16 & 39,0 & $24,0-54,0$ \\
\hline Sem PET/MAH & 25 & 61,0 & $46,0-75,0$ \\
\hline \multicolumn{4}{|c|}{ Retorno à consulta médica $(\mathrm{N}=182)$} \\
\hline Sim & 133 & 73.1 & $20,6-34,0$ \\
\hline Não & 49 & 26,9 & $66,0-79,4$ \\
\hline
\end{tabular}

Dos 182 prontuários de portadores do HTLV-1 envolvidos na pesquisa, $64(35,2 \%)$ eram do sexo masculino, e $118(64,8 \%)$, do sexo feminino. A média \pm desvio padrão e mediana de idade nesses indivíduos foram, respectivamente, $43,78 \pm 12,8$ anos e 44 anos (Tabela 1).

Em relação à situação conjugal dos pacientes consultados, houve o predomínio de indivíduos casados $87(47,8 \%)$ e apenas $6(3,3 \%)$ divorciados. A maioria tinha procedência do município de Belém 134 (73,6\%) e apenas 1 indivíduo (0,5\%) era procedente de outro Estado (Tabela 1).

A condição de trabalhador informal foi predominante nos prontuários pesquisados, com 88 casos $(48,4 \%)$, sendo observados também 14 estudantes $(7,7 \%)$ e 14 aposentados $(7,7 \%)$ como ocupações presentes na história clínica dos pacientes (Tabela 1).

Fato interessante foi observado em relação à sintomatologia desses indivíduos, dos quais 102 (56\%) eram assintomáticos, e 80 (44\%), sintomáticos. Dentre estes últimos, 41 (51\%) apresentavam sintomas neurológicos, 17 (21\%), queixas dermatológicas, 14 (18\%), queixas reumatológicas, e 8 (10\%), queixas autonômicas. Considerando os pacientes com queixas neurológicas, 16 (39,0\%) foram diagnosticados com PET/MAH. Por fim, no que tange ao acompanhamento clínico após a primeira consulta médica, $133(73,1 \%)$ pacientes realizaram acompanhamento, enquanto que $49(26,9 \%)$ não retornaram a nenhuma outra consulta no serviço (Tabela 1).

\section{DISCUSSÃO}

Esta pesquisa consistiu em análise de dados secundários coletados em 182 prontuários de pacientes com HTLV-1 atendidos em um centro público de referência regional em doenças tropicais no Estado do Pará, para caracterização clínica e epidemiológica dos sujeitos.

A faixa etária mais prevalente neste estudo foi de 40 a 49 anos. Dados na literatura relatam que a transmissão via sexual aumenta com o avanço da idade. A relação entre o aumento da idade e a suscetibilidade à infecção, principalmente nas mulheres, pode ser atribuído ao maior número de exposições sexuais com parceiros infectados ao longo da vida, como mostram nos estudos realizados nos Estados do Rio de Janeiro, de Mato Grosso do Sul e de Salvador ${ }^{21-23}$.

Dentre os pacientes infectados pelo HTLV-1, houve o predomínio do sexo feminino. Sequeira et al. ${ }^{24} \mathrm{em}$ seu estudo, no Estado do Pará, sugere que a possível explicação para esse alto índice pode ser dada pela iniciação precoce das relações sexuais e também pelo grande número e variabilidade de parceiros. Outro ponto a ser justificado pela alta prevalência em mulheres está relacionado, segundo Mello et al. ${ }^{25}$, em seu estudo realizado na Bahia, à presença do vírus nas secreções genitais de indivíduos infectados.

Em uma revisão feita por Paiva e Casseb ${ }^{26}$, sugere-se que a prática de atividade sexual desprotegida com parceiros de áreas 
endêmicas é um fator que leva a transmissão do vírus por via sexual, uma vez que a transmissão é mais efetiva do homem para a mulher. No entanto, segundo Lucena et al..$^{27}$ e Giraldo et al. ${ }^{28}$ afirmam que essa infecção pode estar relacionada também com a resposta imunológica celular da vagina, a qual, por meio da ação hormonal estrogênica, sofre modificações epiteliais com maior afluxo de linfócitos locais, favorecendo a formação de um maior número de sinapses celulares, condição necessária para a transmissão intercelular do vírus.

O trabalho informal foi relatado por $48,4 \%$ dos pacientes, o que, de acordo com Ribeiro et al. ${ }^{18}$, Moxoto et al. ${ }^{23}$ e Mello et al. ${ }^{25}$, em seus estudos realizados em Minas Gerais e na Bahia, destaca-se entre indivíduos infectados pelo HTLV-1, associado à baixa escolaridade e ao poder socioeconômico inferior. Isso pode ser explicado pelo fato de esses pacientes não terem acesso às informações necessárias em relação à saúde básica, principalmente no que diz respeito aos mecanismos de prevenção de doenças sexualmente transmissíveis.

Um resultado que chamou atenção foi que a maioria dos pacientes infectados era casada. Apesar de o fator casamento representar, em tese, estabilidade e segurança sexual com manutenção de parceria fixa, uma possível explicação para os altos índices de infecção nesse grupo seria a manutenção de um comportamento de risco dos cônjuges, com intercursos sexuais extraconjugais sem o uso de preservativos. Tal associação já foi investigada por Gabriel et al. ${ }^{29}$, em seu estudo em São Paulo com portadores de HIV, no qual observaram que tanto homens como mulheres demonstravam conhecimento sobre a transmissão do HIV, sabiam as formas de prevenção, entretanto muitas mulheres relatavam saber que seus parceiros mantinham relações extraconjugais rotineiramente e, além de considerarem isso "normal" ou "coisa de homem", não cogitavam a ideia do uso do preservativo entre eles.

A maioria dos sujeitos era procedente do município de Belém-PA ou Região Metropolitana, provavelmente porque a localização do centro de referência local para testagem e acompanhamento clínico dessa população-alvo fique na capital, o que dificulta o deslocamento e acesso de possíveis portadores de HTLV-1 do interior do Estado. Outra explicação seria a utilização do endereço de parentes que residem na Região Metropolitana para fins de cadastro. Porém Monteiro et al.$^{30}$, em seu estudo no Rio de Janeiro, e Catalan-Soares et al. ${ }^{31}$ observaram uma distribuição heterogênea dessa infecção, mostrando que o Estado do Pará é um dos quatro Estados brasileiros com maior prevalência para o HTLV-1 (6,7 a 10 por 1.000/habitantes), junto com os Estados do Maranhão, da Bahia e de Pernambuco. Os demais Estados apresentam uma prevalência abaixo de 3,4/1.000 habitantes, o que reforça o fato de o Estado do Pará ser considerado endêmico, e, portanto, com maior prevalência.

Nos prontuários analisados, foi observado que a maioria era assintomática. Esse resultado pode ser explicado pelo longo tempo de exposição ao vírus, necessário para manifestação da sintomatologia, e que, em alguns casos, muitos portadores permanecem sem apresentar sintomas associados ao HTLV-1 pela vida inteira ${ }^{21}$. Contudo são capazes de transmitir o vírus, desde que o genoma proviral esteja integrado na sequência de DNA da célula hospedeira ${ }^{31}$.

Costa et al..$^{32}$, em seu estudo de transmissão familiar do HTLV realizada no NMT, mostraram que os indivíduos sintomáticos tinham HTLV-1, com sintomas neurológicos em 21 pacientes, manifestações cutâneas em 16, sintomas reumatológicas em 14 e manifestações oculares em 11, o que corrobora os nossos resultados. Apesar de ter sido em uma amostra maior, os sintomas mais prevalentes foram os mesmos.

Dos 41 pacientes com sintomas neurológico, 16 (39,0\%) foram diagnosticados com PET/MAH definitivo ${ }^{20}$, sendo que a outra parcela desses pacientes $(61,0 \%)$ deve ser continuamente monitorada por se tratar de futuros candidatos ao desenvolvimento do complexo neurológico da PET/MAH. Outros sintomas relatados pelos pacientes não podem ser considerados como aleatórios ou sem importância, uma vez que o HTLV-1 promove alteração na resposta imunológica, levando a alterações em diversos outros sistemas. Assim, faz-se necessário o acompanhamento sistemático de todos os pacientes infectados, monitorando qualquer sintoma que pode estar relacionado ao vírus ${ }^{32}$.

No entanto, sabe-se que esse acompanhamento nem sempre ocorre. O resultado da atual pesquisa demonstrou que $26,9 \%$ dos portadores de HTLV-1 pesquisados não retornaram ao ambulatório após a primeira consulta médica para o acompanhamento do seu estado clínico. Muitos não retornam provavelmente por não manifestarem sintomas graves ou incapacitantes no momento do diagnóstico da infecção viral, considerando sem importância o seu acompanhamento clínico regular. Porém, quando retornam ao serviço, é porque eles já estão em uma condição clínica grave, apresentando incapacidades físicas, o que gera um manejo maior no seu tratamento. Esse momento em que esses pacientes estão no limbo do acompanhamento clínico é ponto crucial para o diagnóstico precoce de qualquer alteração que esse indivíduo possa apresentar.

\section{CONCLUSÃO}

Os resultados deste estudo confirmaram dados de um perfil epidemiológico muito próximo do que é encontrado na literatura. Sobre os aspectos gerais que caracterizaram o grupo, conforme a análise descritiva, destacam-se o predomínio do sexo feminino, a faixa etária entre 40 e 49 anos, o estado civil casado, o trabalho informal, a procedência de Belém-PA, as queixas neurológicas e o retorno às consultas médicas pela grande maioria dos pacientes.

Acredita-se que estudos dessa natureza podem fornecer dados mais específicos e direcionados acerca da realidade clínica e epidemiológica local dos portadores de HTLV-1, o que pode subsidiar a elaboração de estratégias regionalizadas de manejo clínico e de reabilitação desses pacientes. 


\section{REFERÊNCIAS}

1. Cooper SA, van der Loeff MS, Taylor GP. The neurology of HTLV-1 infection. Pract Neurol. 2009;9(1):16-26. http://dx.doi.org/10.1136/jnnp.2008.167155. PMid:19151234.

2. Gessain A, Mahieux R. Tropical spastic paraparesis and HTLV-1 associated myelopathy: clinical, epidemiological, virological and therapeutic aspects. Rev Neurol (Paris). 2012;168(3):257-69. http://dx.doi.org/10.1016/j. neurol.2011.12.006. PMid:22405461.

3. Lannes P, Neves MAO, Machado DCD, Miana LC, Silva JG, Bastos VHV. Paraparesia Espástica Tropical - Mielopatia associada ao vírus HTLV-I: possíveis estratégias cinesioterapêuticas para a melhora dos padrões de marcha em portadores sintomáticos. Rev Neurocienc. 2006;14(3):153-60.

4. Verdonck K, González E, Van Dooren S, Vandamme AM, Vanham G, Gotuzzo E. Human T-lymphotropic virus 1: recent knowledge about an ancient infection. Lancet Infect Dis. 2007;7(4):266-81. http://dx.doi. org/10.1016/S1473-3099(07)70081-6. PMid:17376384.

5. Yamano Y, Araya N, Sato T, Utsunomiya A, Azakami K, Hasegawa D, et al. Abnormally high levels of virus-infected IFN-gamma+ CCR4+ CD4+ $\mathrm{CD} 25+\mathrm{T}$ cells in a retrovirus-associated neuroinflammatory disorder. PLoS One. 2009;4(8):e6517. http://dx.doi.org/10.1371/journal.pone.0006517. PMid:19654865.

6. Romanelli LC, Caramelli P, Proietti AB. Human T cell lymphotropic virus (HTLV-1): when to suspect infection?. Rev Assoc Med Bras. 2010;56(3):3407. http://dx.doi.org/10.1590/S0104-42302010000300021. PMid:20676544.

7. Matsuura E, Yamano Y, Jacobson S. Neuroimmunity of HTLV-I Infection. J Neuroimmune Pharmacol. 2010;5(3):310-25. http://dx.doi.org/10.1007/ s11481-010-9216-9. PMid:20437106.

8. Olière S, Douville R, Sze A, Belgnaoui SM, Hiscott J. Modulation of innate immune responses during human T-cell leukemia virus (HTLV-1) pathogenesis. Cytokine Growth Factor Rev. 2011;22(4):197-210. http:// dx.doi.org/10.1016/j.cytogfr.2011.08.002. PMid:21924945.

9. Saito M. Immunogenetics and the Pathological Mechanisms of Human T-Cell Leukemia VirusType 1- (HTLV-1) Associated Myelopathy/Tropical Spastic Paraparesis (HAM/TSP). Interdiscip Perspect Infect Dis. 2010;2010:478461. http://dx.doi.org/10.1155/2010/478461. PMid:20169122.

10. Sonoda S, Li HC, Tajima K. Ethnoepidemiology of HTLV-1 related diseases: ethnic determinants of HTLV-1 susceptibility and its worldwide dispersal. Cancer Sci. 2011;102(2):295-301. http://dx.doi.org/10.1111/j.13497006.2010.01820.x. PMid:21205073.

11. Watanabe T. Current status of HTLV-1 infection. Int J Hematol. 2011;94(5):4304. http://dx.doi.org/10.1007/s12185-011-0934-4. PMid:21969187.

12. Dourado I, Alcantara LC, Barreto ML, Teixeira MG, Galvão-Castro B. HTLV-I in the general population of Salvador, Brazil: a city with African ethnic and sociodemographic characteristics. J Acquir Immune Defic Syndr. 2003;34(5):527-31. http://dx.doi.org/10.1097/00126334-200312150-00013. PMid:14657765.

13. Jacob F, Santos-Fortuna EL, Azevedo RS, Caterino-de-Araujo A. Performances of HTLV serological tests in diagnosing HTLV infection in high-risk population of São Paulo, Brazil. Rev Inst Med Trop Sao Paulo. 2007;49(6):361-4. http://dx.doi.org/10.1590/S0036-46652007000600005. PMid:18157402.

14. Silva CMS, Sousa VG, Pires C, Martins CS, Gomes SCS, Mochel EG. Prevalência de sorologia positiva para o HTLV-1 e HTLV-2 em gestantes atendidas em três serviços de pré-natal, São Luis, jul/08 a jul/09. Cad Pesq São Luis. 2009;16(3):39-44.

15. Champs AP, Passos VM, Barreto SM, Vaz LS, Ribas JG. Mielopatia associada ao HTLV-1: análise clínico-epidemiológica em uma série de casos de 10 anos. Rev Soc Bras Med Trop. 2010;43(6):668-72. http://dx.doi.org/10.1590/ S0037-86822010000600013. PMid:21181020.

16. Galvão-Castro B, Loures L, Rodriques LG, Sereno A, Ferreira Júnior OC, Franco LG, et al. Distribution of human T-lymphotropic virus type I among blood donors: a nationwide Brazilian study. Transfusion. 1997;37(2):242-3. http://dx.doi.org/10.1046/j.1537-2995.1997.37297203532.x. PMid:9051104.

17. Lairmore MD, Haines R, Anupam R. Mechanisms of human T-lymphotropic virus type 1 transmission and disease. Curr Opin Virol. 2012;2(4):474-81. http://dx.doi.org/10.1016/j.coviro.2012.06.007. PMid:22819021.

18. Ribeiro MA, Proietti FA, Martins ML, Januário JN, Ladeira RV, Oliveira MF, et al. Geographic distribution of human T-lymphotropic virus types 1 and 2 among mothers of newborns tested during neonatal screening, Minas Gerais, Brazil. Rev Panam Salud Publica. 2010;27(5):330-7. http:// dx.doi.org/10.1590/S1020-49892010000500002. PMid:20602066.

19. Santos FLN, Lima FWM. Epidemiologia, fisiopatogenia e diagnóstico laboratorial da infecção pelo HTLV-I. J Bras Patol Med Lab. 2005;41(2):10516.

20. Castro-Costa CM, Araújo AQ, Barreto MM, Takayanagui OM, Sohler MP, Silva EL, et al. Proposal for diagnostic criteria of tropical spastic paraparesis/HTLV-I-associated myelopathy (TSP/HAM). AIDS Res Hum Retroviruses. 2006;22(10):931-5. http://dx.doi.org/10.1089/aid.2006.22.931. PMid:17067261.

21. Franzoi AC, Araújo AQC. Disability profile of patients with HTLV-Iassociated myelopathy/tropical spastic paraparesis using the Functional Independence Measure (FIM). Spinal Cord. 2005;43(4):236-40. http:// dx.doi.org/10.1038/sj.sc.3101677. PMid:15520834.

22. Dal Fabbro MM, Cunha RV, Bóia MN, Portela P, Botelho CA, Freitas GM, et al. HTLV 1/2 infection: prenatal performance as a disease control strategy in State of Mato Grosso do Sul. Rev Soc Bras Med Trop. 2008;41(2):148-51. PMid:18545834.

23. Moxoto I, Boa-Sorte N, Nunes C, Mota A, Dumas A, Dourado I, et al Sociodemographic, epidemiological and behavioral profile of women infected with HTLV-1 in Salvador, Bahia, an endemic area for HTLV. Rev Soc Bras Med Trop. 2007;40(1):37-41. http://dx.doi.org/10.1590/S003786822007000100007. PMid:17486251

24. Sequeira CG, Tamegão-Lopes BP, Santos EJ, Ventura AM, Moraes-Pinto MI, Succi RC. Descriptive study of HTLV infection in a population of pregnant women from the state of Pará, Northern Brazil. Rev Soc Bras Med Trop. 2012;45(4):453-6. http://dx.doi.org/10.1590/S0037-86822012005000007. PMid:22836660.

25. Mello MA, Conceição AF, Sousa SMB, Alcântara LC, Marin LJ, Raio MRS, et al. HTLV-1 in pregnant women from the Southern Bahia, Brazil: a neglected condition despite the high prevalence. Virol J. 2014;11(1):28. http://dx.doi.org/10.1186/1743-422X-11-28. PMid:24524416.

26. Paiva A, Casseb J. Sexual transmission of human T-cell lymphotropic virus type 1. Rev Soc Bras Med Trop. 2014;47(3):265-74. http://dx.doi. org/10.1590/0037-8682-0232-2013. PMid:25075475.

27. Lucena AAS, Michelin MA, Guimarães MVMB, Lodi CTC, Miranda MIL, Murta EFC, et al. Resposta imune celular ao papilomavírus humano 
em mulheres infectadas e não infectadas pelo vírus da imunodeficiência humana. Femina. 2011;39(3):150-5

28. Giraldo PC, Feitoza SBN, Gonçalves AKS, Cornetta MCM, Eleutério Jr J, Tristão AR. A resposta imune celular da mucosa vaginal às vulvovaginites. J Bras Doenças Sex Transm. 2006;18(4):263-65.

29. Gabriel R, Barbosa DA, Vianna LAC. Perfil epidemiológico dos clientes com HIV/AIDS da unidade ambulatorial de hospital escola de grande porte - município de São Paulo. Rev Latino-am Enfermagem. 2005;13(4):509-13. http://dx.doi.org/10.1590/S0104-11692005000400008.

30. Monteiro DL, Taquette SR, Sodré Barmpas DB, Rodrigues NC, Teixeira SA, Villela LH, et al. Prevalence of HTLV-1/2 in pregnant women living in the metropolitan area of Rio de Janeiro. PLoS Negl Trop Dis. 2014;8(9):e3146. http://dx.doi.org/10.1371/journal.pntd.0003146. PMid:25188386.
31. Catalan-Soares B, Carneiro-Proietti AB, Proietti FA, Interdisciplinary HTLV Research Group. Heterogeneous geographic distribution of human T-cell lymphotropic viruses I and II (HTLV-I/II): serological screening prevalence rates in blood donors from large urban areas in Brazil. Cad Saude Publica. 2005;21(3):926-31. http://dx.doi.org/10.1590/S0102-311X2005000300027. PMid:15868051.

32. Costa CA, Furtado KC, Ferreira LS, Almeida DS, Linhares AC, Ishak $\mathrm{R}$, et al. Familial transmission of human T-cell lymphotrophic virus: silent dissemination of an emerging but neglected infection. PLoS Negl Trop Dis. 2013;7(6):e2272. http://dx.doi.org/10.1371/journal.pntd.0002272. PMid:23785534.

Recebido em: Dez. 01, 2014 Aprovado em: Jul. 13, 2015 